\title{
Effect of phase-plate adjustment on retinal image sharpness and visible retinal area on ultrawide field imaging
}

\author{
Aditi Gupta ${ }^{1}{ }^{1}$ - Hala El-Rami ${ }^{1}$ - Rasha Barham ${ }^{1} \cdot$ Alan Fleming $^{2}$ - Jano van Hemert ${ }^{2}$ Jennifer K. Sun ${ }^{1,3}$. \\ Paolo S. Silva ${ }^{1,3,4} \cdot$ Lloyd Paul Aiello ${ }^{1,3}$
}

Received: 9 April 2018 / Revised: 12 July 2018 / Accepted: 13 September 2018 / Published online: 2 November 2018

(c) The Royal College of Ophthalmologists 2018

\begin{abstract}
Background To evaluate changes in image sharpness across ultrawide field (UWF) images and the effect of phase-plate adjustment on image contrast and extent of visible retinal area (VRA).

Methods This was a single site evaluation of $200^{\circ}$ UWF images acquired with phase-plate adjustment (California, Optos, plc) and without (200TX, Optos, plc). Images were acquired using standardized protocol. VRA was manually outlined on each image and quantified using customized software. Mean image sharpness was evaluated using an automated method within the full VRA of each image and within the peripheral region of the VRA. The VRA and image sharpness were evaluated and compared between the two devices.

Results Twenty eyes of 10 healthy volunteers were evaluated. Devices with and without phase-plate adjustment produced a similar extent of VRA. Eye steering increased VRA in devices with and without phase-plate adjustment by $39.3 \%$ and $34.3 \%$, respectively. Regardless of gaze direction, mean sharpness of the full VRA was reduced in peripheral area with or without phase-plate adjustment. Compared to images without phase-plate adjustment, use of phase-plate adjustment reduced the loss of peripheral image sharpness in all fields ( -4.2 to $-26.0 \% ; p<0.001$ all fields). The sharpness of the peripheral area for on-axis images was $61.5 \%$ higher with phase-plate adjustment.

Conclusions The use of phase-plate adjustment does not alter the extent of VRA. However, for on-axis images the loss of sharpness in the periphery is 4.5 -fold less with phase-plate adjustment, potentially reducing the need to steer images and improving lesion detection in these areas.
\end{abstract}

\section{Introduction}

The ultrawide field (UWF) confocal scanning laser ophthalmoscope allows fast, noncontact imaging from the posterior pole to the peripheral retina in a single image. It is now used for numerous retinal applications in the fields of diabetic retinopathy [1], macular degeneration [2], posterior

Paolo S. Silva

paoloantonio.silva@joslin.harvard.edu

Beetham Eye Institute, Joslin Diabetes Center, Boston, MA, USA

2 Optos, plc, Dunfermline, Scotland, UK

3 Department of Ophthalmology, Harvard Medical School, Boston, MA, USA

4 Teleophthalmology and Image Reading Center, Philippine Eye Research Institute, National Institutes of Health, University of the Philippines, Manila, Philippines uveitis [3], and teleophthalmology [4, 5]. With UWF imaging, the retinal surface is scanned using two lasers that are reflected off an ellipsoid mirror. The resulting image encompasses $892.3 \mathrm{~mm}^{2}$ of visible retinal area (VRA) through an undilated pupil, corresponding to $\sim 200^{\circ}$ or $82.5 \%$ of the retinal surface. The distortion in the VRA and loss of image sharpness in the peripheral retina are inherent with the use of UWF retinal imaging due to flattening of the three-dimensional spherical shape of the eye to generate a two-dimensional image [6]. Compared to retinal imaging utilizing $30-50^{\circ}$ fields, the peripheral distortion is more noticeable with UWF imaging due to the much larger VRA imaged. This distortion is further pronounced by the use of an ellipsoid mirror resulting in an image that appears particular distorted in the far temporal and nasal periphery. To further increase the amount of peripheral field acquired, the axis can be shifted by 'steering' the location during image acquisition thereby allowing observation of more peripheral portions of the retina. The steered images have been shown 
to have a decrease in image quality secondary to decreasing width of the entrance pupil and vignetting [7]. The latest commercially available UWF device (California, Optos plc, Dunfermline, UK) incorporates a phase-plate adjustment in an attempt to correct for peripheral distortion.

The use of the adjustable phase-plate introduces a novel optical design for UWF imaging that theoretically can produce sharp, in-focus imaging over the entire retina with less blurring of peripheral retina [8]. The phase-plate is a static aberration correction element, whose location maintains the transfer of the beam of light from the apparent point source at the pupillary plane of the eye to the retina. The purpose of the present study is to evaluate the extent of image quality loss in the periphery of UWF imaging and the effect of phase-plate adjustment. For that purpose, we have compared similar devices with and without the correction element and determined the effect on image contrast (sharpness) and VRA.

\section{Subjects and methods}

Twenty eyes of 10 healthy volunteers were evaluated. Exclusion criteria included presence of any ocular disease. Demographic data, the eye's refractive power (in form of spherical equivalent) and axial length were recorded. For each study eye, UWF imaging with the California (Optos plc, Dunfermline, UK), which utilizes phase-plate adjustment element, and 200TX (Optos plc, Dunfermline, UK), which does not utilize phase-plate adjustment element, were acquired using a standardized protocol in a single sitting at the same visit. Images were acquired by clinical trial certified retinal imagers participating in multicenter UWF imaging studies. The protocol consisted of obtaining two stereoscopic on-axis images (a stereo pair), and one each for monoscopic steered nasal, temporal, inferior, and superior fields.

\section{Evaluation of visible retinal area}

For each eye, the foveal location was manually annotated on each UWF image. The VRA was manually delineated by drawing around the visible retina on each image after adjusting brightness and contrast for an optimal view of the retina. General stereographic projection was applied to all images using the manually annotated fovea position to correct for image distortion. The same projection was applied to the manually annotated VRA. VRA was then quantitated using customized software provided by the manufacturer (Optos plc, Dunfermline, UK) as area in mm [2]. Gaze direction was determined from the manually annotated fovea location. VRA in pairs of corresponding images from the same eye and gaze direction but different devices were compared. To allow straightforward comparisons of images between devices, where more than one image was present for the same eye, same gaze direction and same device, the image with the greatest VRA was selected. The manually outlined VRAs of the on-axis and steered images were montage for each eye and the total VRA in $\mathrm{mm}^{2}$ was calculated. All retinal area calculations were corrected for the individual eye's axial length.

\section{Evaluation of image contrast}

Due to a fundamental difference between pixel value histograms of the Optos California and 200TX images, a gamma correction was applied to the 200TX images to simulate the gamma correction made to California images in the image capture software. The mean image sharpness (expressed as mean absolute percentage change in brightness per pixel after low pass filtering) was evaluated using an automated method within the full VRA of each stereographically projected image and within the peripheral region of the VRA located anterior to a circle of 4.5 discdiameter radius centered on the fovea. Sharpness was compared in corresponding regions in the same pairs of images (same eye, same gaze direction, different devices) that were used for the VRA comparison.

\section{Results}

Mean age of 10 volunteers was $37.50 \pm 16.25$ years (range 23-68), refractive error (spherical equivalent) was $-1.71 \pm$ 2.89 diopters (range -7.50 to +3.0 ) and axial length was $24.09 \pm 1.24 \mathrm{~mm}$ (range 22.47-26.88). The VRA in each gaze direction is shown in Table 1. There were no statistically significant differences in VRA between the two devices regardless of gaze direction other than for superior steered images. In superior steered images, phase-plate adjusted imaging provided a larger VRA of $607.8 \mathrm{~mm}^{2}$ vs $552.3 \mathrm{~mm}^{2}$ without phase-plate adjustment (difference of $9.9 \%, p=0.002$ ). Compared to on-axis imaging alone, eye steering allowed visualization of additional retinal area for both devices (39.3\% with phase-plate adjustment and $34.3 \%$ without, $p=0.33$ ). A comparison of stereographic projected UWF images with and without phase-plate adjustment are shown in Fig. 1.

The mean image sharpness of the full VRA and of the peripheral retinal area for on-axis and steered images are shown in Table 2. The loss of sharpness between the peripheral VRA and the full VRA was statistically significant for all images acquired either with phase-plate adjustment $(-7.0 \%, p<0.001)$ or without $(-31.8 \%, p<0.001)$. However, the use of phase-plate adjustment reduced the loss of peripheral image sharpness in all fields by $9.8-25.1 \%$ 
Table 1 Visible retinal area obtained with phase-plate adjustment and without phase-plate adjustment

\begin{tabular}{lllllll}
\hline Visible retinal area $\left(\mathrm{mm}^{2}\right)$ & On-axis & Nasal & Temporal & Inferior & Superior & Total \\
\hline Phase-plate adjustment & $673.3 \pm 26.1$ & $596.2 \pm 24.8$ & $590.6 \pm 20.7$ & $602.4 \pm 24.8$ & $607.8 \pm 24.8$ & $921.7 \pm 24.7$ \\
Without phase-plate adjustment & $683.4 \pm 22.5$ & $578.3 \pm 20.9$ & $551.3 \pm 16.9$ & $618.7 \pm 18.6$ & $552.3 \pm 17.1$ & $908.0 \pm 21.5$ \\
Difference with phase-plate adjustment (\%) & $-1.9 \pm 3.0$ & $3.5 \pm 3.1$ & $6.7 \pm 3.2$ & $-0.8 \pm 3.5$ & $9.9 \pm 2.7$ & $1.8 \pm 2.1$ \\
p-Value & 0.43 & 0.32 & 0.07 & 0.64 & 0.002 \\
\hline
\end{tabular}

Data presented as mean \pm standard error. $p$-Value was determined using $t$-test.

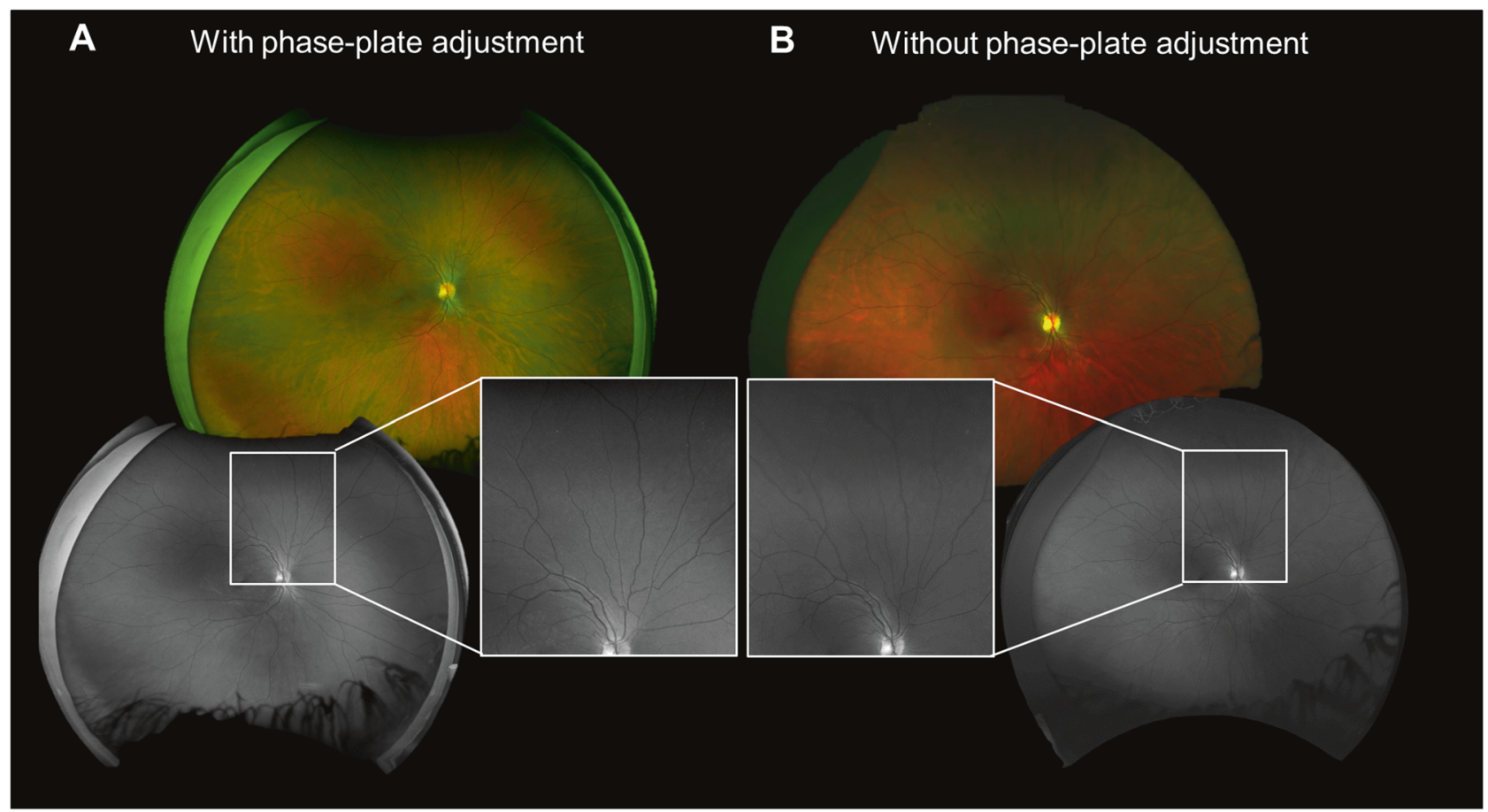

Fig. 1 Shows stereographically projected ultrawide retinal on-axis images with (a) and without (b) phase-plate adjustment. (color composite images, top; green separation/red free images, bottom). Inset shows the magnified view of the area outlined by the white rectangle

$(p<0.001)$. This was most marked in on-axis and nasal steered images with sharpness difference improvements of $25.1 \%$ and $26.0 \%$, respectively.

\section{Discussion}

Ultrawide field imaging is becoming more prevalent and provides numerous advantages over traditional ETDRS 7standard field photography, especially in the evaluation and care of diabetic retinopathy [9-13]. However, previous UWF image capture devices have suffered from substantially diminished peripheral sharpness, especially superiorly and inferiorly. Since it has been shown that peripheral diabetic retinal lesions predict an increased risk of diabetic retinopathy progression and onset of proliferative diabetic eye disease $[14,15]$, it is critical that image quality is optimized throughout the periphery to best evaluate subtle lesions of diabetic eye disease. The detailed evaluation of the peripheral retina may help more accurately assess risk of diabetic retinopathy severity progression [1].

Previous work has shown that using pharmacologic mydriasis with UWF did not result in a statistically significant increase in the over-all agreement with 7-standard field Early Treatment Diabetic Retinopathy Study (ETDRS) stereoscopic $35 \mathrm{~mm}$ film [1, 16]. However, mydriasis reduced the ungradable rate of UWF images from $4.5 \%$ (9 eyes) to $0 \%(p=0.002)$. Furthermore, mydriatic UWF imaging improved the individual lesion agreement with ETDRS film as compared to nonmydriatic imaging [1]. Using UWF imaging without phase-plate adjustment, the ungradable rates for peripheral lesions were twofold greater in the inferior temporal field and threefold greater in the inferior nasal field as compared to the temporal, superior temporal and superior nasal fields [1]. The suboptimal image quality and higher ungradable rates in the inferior 
Table 2 Comparison of image sharpness by field of view

\begin{tabular}{|c|c|c|c|c|c|c|}
\hline Phase-plate adjustment & Area & On-axis & Nasal & Temporal & Inferior & Superior \\
\hline \multirow[t]{4}{*}{ Phase-plate adjustment } & Full & $1.07 \pm 0.03$ & $1.09 \pm 0.03$ & $0.99 \pm 0.04$ & $1.09 \pm 0.06$ & $0.97 \pm 0.03$ \\
\hline & Peripheral & $0.99 \pm 0.03$ & $1.02 \pm 0.03$ & $0.93 \pm 0.04$ & $0.98 \pm 0.05$ & $0.88 \pm 0.03$ \\
\hline & Full-peripheral difference $(\%)$ & $-7.0 \pm 0.5$ & $-6.9 \pm 0.5$ & $-6.0 \pm 1.5$ & $-10.5 \pm 0.6$ & $-10.0 \pm 0.7$ \\
\hline & $p$-Value (full vs peripheral) & $<0.001$ & $<0.001$ & $<0.001$ & $<0.001$ & $<0.001$ \\
\hline \multirow[t]{4}{*}{ Without phase-plate adjustment } & Full & $0.89 \pm 0.05$ & $0.90 \pm 0.05$ & $1.38 \pm 0.08$ & $0.94 \pm 0.05$ & $1.25 \pm 0.06$ \\
\hline & Peripheral & $0.613 \pm 0.04$ & $0.610 \pm 0.04$ & $1.124 \pm 0.10$ & $0.761 \pm 0.05$ & $1.083 \pm 0.06$ \\
\hline & Full-peripheral difference (\%) & $-31.8 \pm 1.3$ & $-32.7 \pm 1.5$ & $-20.7 \pm 2.7$ & $-20.2 \pm 1.9$ & $-14.2 \pm 1.3$ \\
\hline & $p$-Value (full vs peripheral) & $<0.001$ & $<0.001$ & $<0.001$ & $<0.001$ & $<0.001$ \\
\hline \multicolumn{2}{|c|}{$\begin{array}{l}\text { Increase in "Full-peripheral difference" phase-plate adjustment } \\
\text { included }(\%)\end{array}$} & $25.1 \pm 1.4$ & $26.0 \pm 1.5$ & $15.6 \pm 1.9$ & $9.8 \pm 1.8$ & $4.2 \pm 1.2$ \\
\hline \multicolumn{2}{|c|}{$\begin{array}{l}p \text {-Value (full-peripheral difference, phase-plate vs without phase- } \\
\text { plate }\end{array}$} & $<0.001$ & $<0.001$ & $<0.001$ & $<0.001$ & $<0.001$ \\
\hline
\end{tabular}

Data presented as means or $\% \pm$ standard error. $p$-Value was determined using $t$-test.

and superior periphery highlights the need for improved image acquisition such as with phase-plate adjustment.

With the implementation of phase-plate adjustment, the loss of image sharpness in the periphery was markedly reduced in both the posterior pole and all peripheral quadrants. The loss of sharpness in the image periphery for onaxis images was 4.5-fold less with phase-plate adjustment potentially reducing the need to steer images and improving lesion detection in the peripheral retina. However, the use of phase-plate adjustment does not alter the extent of VRA. The degree to which better peripheral image sharpness facilitates identification of individual retinal lesions in the retinal periphery without the added time, patient discomfort and imager effort required to attain steered images remains to be determined.

\section{Summary}

\section{What was known before}

- Peripheral image sharpness is substantially reduced compared with posterior sharpness in ultrawide field retinal images.

\section{What this study adds}

- Although phase-plate adjustment does not alter the extent of visible retinal area, there is 4.5 -fold less loss of sharpness in the periphery compared to imaging without phase-plate adjustment.

- Phase-plate adjustment on ultrawide field imaging increases peripheral image sharpness that may potentially allow better identification of peripheral retinal lesions.
Acknowledgements This study was supported by the Massachusetts Lions Eye Research Fund (Aiello). None of the sources of funding support had any role in the design and conduct of the study; collection, management, analysis, and interpretation of the data; preparation, review, or approval of the manuscript; and decision to submit the manuscript for publication.

Author contributions AG and PSS had full access to all the data in the report and takes responsibility for the integrity of the data and the accuracy of the information presented. Study concept and design: AG, PSS, and LPA. Acquisition, analysis, or interpretation of data: all authors. Drafting of the manuscript: PSS, AG, and LPA. Critical revision of the manuscript for important intellectual content: all authors. Obtained funding: PSS and LPA. Administrative, technical, or material support: PSS and LPA. Study supervision: PSS and LPA.

\section{Compliance with ethical standards}

Conflict of interest Temporary loan of two ultrawide-field imaging devices was provided to the Joslin Diabetes Center (Optos plc, Dunfermline, Scotland).

\section{References}

1. Silva PS, Cavallerano JD, Sun JK, Soliman AZ, Aiello LM, Aiello LP. Peripheral lesions identified by mydriatic ultrawide field imaging: distribution and potential impact on diabetic retinopathy severity. Ophthalmology. 2013;120:2587-95.

2. Tan CS, Heussen F, Sadda SR. Peripheral autofluorescence and clinical findings in neovascular and non-neovascular age-related macular degeneration. Ophthalmology. 2013;120:1271-7.

3. Kaines A, Tsui I, Sarraf D, Schwartz S. The use of ultra wide field fluorescein angiography in evaluation and management of uveitis. Semin Ophthalmol. 2009;24:19-24.

4. Silva PS, Cavallerano JD, Tolls D, et al. Potential efficiency benefits of nonmydriatic ultrawide field retinal imaging in an ocular telehealth diabetic retinopathy program. Diabetes Care. 2014;37:50-55.

5. Kernt M, Hadi I, Pinter F, et al. Assessment of diabetic retinopathy using nonmydriatic ultra-widefield scanning laser 
ophthalmoscopy (Optomap) compared with ETDRS 7-field stereo photography. Diabetes Care. 2012;35:2459-63.

6. Sagong M, van HJ, Olmos de Koo LC, Barnett C, Sadda SR. Assessment of accuracy and precision of quantification of ultrawidefield images. Ophthalmology. 2015;122:864-6.

7. Oishi A, Hidaka J, Yoshimura N. Quantification of the image obtained with a wide-field scanning ophthalmoscope. Invest Ophthalmol Vis Sci. 2014;55:2424-31.

8. Sadda SR, Sagong M, van Hemert J. Comparison of ultrawidefield Optos imaging with and without phase mask implementation. Invest Ophthalmol \& Vis Sci. 2015;56:1433-1433.

9. Tan CS, Sadda SR, Hariprasad SM. Ultra-widefield retinal imaging in the management of diabetic eye diseases. Ophthalmic Surg Lasers Imaging Retin. 2014;45:363-6.

10. Kiss S, Berenberg TL. Ultra widefield fundus imaging for diabetic retinopathy. Curr Diab Rep. 2014;14:514.

11. Nagiel A, Lalane RA, Sadda SR, Schwartz SD. Ultra-widefield fundus imaging: a review of clinical applications and future trends. Retina. 2016;36:660-78.

12. Silva PS, Walia S, Cavallerano JD, et al. Comparison of low-light nonmydriatic digital imaging with 35-mm ETDRS seven-standard field stereo color fundus photographs and clinical examination. Telemed J E Health. 2012;18:492-9.

13. Silva PS, Cavallerano JD, Sun JK, Noble J, Aiello LM, Aiello LP. Nonmydriatic ultrawide field retinal imaging compared with dilated standard 7-field 35-mm photography and retinal specialist examination for evaluation of diabetic retinopathy. Am J Ophthalmol. 2012;154:549-59 e542.

14. Silva PS, Cavallerano JD, Haddad NM, et al. Peripheral lesions identified on ultrawide field imaging predict increased risk of diabetic retinopathy progression over 4 years. Ophthalmology. 2015;122:949-56.

15. Silva PS, Dela Cruz AJ, Ledesma MG, et al. Diabetic retinopathy severity and peripheral lesions are associated with nonperfusion on ultrawide field angiography. Ophthalmology. 2015;122:246572.

16. Silva PS, Cavallerano JD, Sun JK, Noble J, Aiello LM, Aiello LP. Nonmydriatic ultrawide field retinal imaging compared with dilated standard 7-field 35-mm photography and retinal specialist examination for evaluation of diabetic retinopathy. Am J Ophthalmol. 2012;154:549-59. 